\title{
Benefit of adjuvant chemoradiotherapy in patients with pathological stage III gastric cancer
}

This article was published in the following Dove Press journal:

Cancer Management and Research

\author{
Gui-Fen Ma',* \\ Hai-Ge Zhang ${ }^{1} * *$ \\ Juan Liu' \\ Yi-Xing Chen' \\ Han Xiao' \\ Xue-Fei Wang ${ }^{2}$ \\ Jian $\mathrm{He}^{\prime}$ \\ Zhao-Chong Zeng' \\ Jing Sun' \\ Tian-Shu Liu ${ }^{3,4}$ \\ 'Department of Radiotherapy, \\ Zhongshan Hospital, Fudan University, \\ Shanghai 200032, People's Republic of \\ China; ${ }^{2}$ Department of General Surgery, \\ Zhongshan Hospital, Fudan University, \\ Shanghai 200032, People's Republic of \\ China; ${ }^{3}$ Department of Medical \\ Oncology, Zhongshan Hospital, Fudan \\ University, Shanghai 200032, People's \\ Republic of China; ${ }^{4}$ Center of Evidence- \\ Based Medicine, Fudan University, \\ Shanghai 200032, People's Republic of \\ China \\ *These authors contributed equally to \\ this work
}

\begin{abstract}
Background: For patients with locally advanced gastric cancer (LAGC) after D2 gastrectomy, the survival benefits of receiving adjuvant chemoradiotherapy versus adjuvant chemotherapy are unclear. This study aimed to compare the 5- and 7-year overall survival (OS) in the two groups and to identify which patients can benefit more from adjuvant chemoradiotherapy.
\end{abstract}

Methods: Retrospective data were collected from January 2009 to December 2014. The 5- and 7 -year OS and disease-free survival (DFS) were compared between the two groups using the Chi-square test. The association of OS with prognostic factors was identified using the Cox's proportional hazard model, which was then adjusted for survival coparison using propensity score-matching (PSM) analysis. The association of OS with each clinical/demographic factor was compared between the two groups using the Kaplan-Meier analysis.

Results: A total of 415 eligible patients were identified (135 adjuvant chemoradiotherapy, 280 adjuvant chemotherapy). Significant 5- and 7-year OS and DFS benefits were found in the adjuvant chemoradiotherapy group versus chemotherapy group. Multivariate analysis showed that age, TNM stage, lymph node (LN) ratio, tumor deposits, and total/subtotal gastrectomy were independent prognostic factors. When the PSM analysis was adjusting by these factors, 135 patients were matched with an improved survival benefit from adjuvant chemoradiotherapy. Patients in the adjuvant chemoradiotherapy group had a lower locoregional relapse. Subset analysis also identified significant OS benefits of adjuvant chemoradiotherapy in patients with $\mathrm{LN}$ ratio $<50 \%$, pIIIA, and pIIIB stage disease, while OS benefits were not observed in patients with tumor deposits, pN3b classification, or pIIIC stage disease.

Conclusion: Adjuvant chemoradiotherapy was shown to be superior in improving the OS in a certain population of patients compared with adjuvant chemotherapy. This finding may help to better guide the individualized treatments of patients with stage III LAGC after D2 gastrectomy. Keywords: adjuvant chemoradiotherapy, adjuvant chemotherapy, local advanced gastric cancer, overall survival, propensity score-matching analysis, tumor deposits

\section{Introduction}

Gastric cancer is a prevalent malignancy worldwide, particularly in Asia. According to the latest cancer statistics, China ranked fourth in terms of gastric cancer incidence (up to 679.1 per 100,000) and third in terms of gastric cancer-related deaths. ${ }^{1}$ The primary treatment for locally advanced gastric cancer (LAGC) is surgical resection. However, the role of chemoradiotherapy in patients with LAGC has been controversial.

Since the publication of the results from the ACTS-GC and CLASSIC trials, ${ }^{2,3}$ adjuvant chemotherapy after D2 gastrectomy has become popular in the East, as such patients demonstrated an improved overall survival (OS) against those having surgery
Department of Radiotherapy, Zhongshan Hospital, Fudan University, I80 Fenglin Road, Shanghai 200032, People's Republic of China

Tel +862II 3795244437

Email sun.jing@zs-hospital.sh.cn

Tian-Shu Liu

Department of Medical Oncology,

Zhongshan Hospital, Fudan University,

Shanghai 200032, People's Republic of China

Email liu.tianshu@zs-hospital.sh.cn 
alone, especially for patients with stage II, IIIA, or IIIB gastric cancer. However, in the West, intensive perioperative chemotherapy and postoperative chemoradiotherapy are recommended for resectable LAGC, since their SWOG/ INT-0116 trial showed that the OS and recurrence-free survival(RFS) of gastric cancer patients who have had R0 resection followed by adjuvant chemoradiotherapy (45 Gy of radiotherapy combined with bolus fluorouracil [FU] and leucovorin) were significantly better to those who had surgery alone (5-year OS, $40 \%$ vs $30 \%$, respectively; 5 -year RFS, $48 \%$ vs $31 \%$, respectively). ${ }^{4}$ Further, for R0 resected patients with stage pT3-4NxM0 or pTxN+M0, the National Comprehensive Cancer Network (NCCN) guideline recommends prescribing fluoropyrimidine-based chemoradiotherapy if they underwent a D1 gastrectomy or did not undergo any lymphadenectomy (category I), or chemotherapy for those who have had undergone a D2 gastrectomy (category I), but it also recommends chemoradiotherapy for high-risk patients after D2 gastrectomy, that is for those with poorly differentiated or higher grade cancer, having lymphovascular and/or neural invasion, or age $<50$ years. ${ }^{5}$

As such, the types of adjuvant therapy to prescribe for LAGC and which subgroups of LAGC patients will optimally benefit from which type of adjuvant therapy remain a major challenge in health care systems throughout the world. According to the results of the ACTS-GC trial, patients with stage IIIA and IIIB gastric cancer had a lower survival time than those with stage II gastric cancer treated with surgery alone (5-year OS rate: $57.3 \%, 44.1 \%$, and 71.3 , respectively). ${ }^{2}$ In China, most patients are diagnosed with stage III at their initial outpatient visit, who are supposed to have a poor survival; therefore, a more aggressive clinical management may be required for them. An analysis from the US National Cancer Database found that for patients with LAGC, adjuvant chemoradiotherapy was associated with a significant OS advantage as compared with chemotherapy; however, only a small percentage of the cohort (17.9\%) had stage III disease. ${ }^{6}$ Similarly, the Korean ARTIST trial demonstrated that for stage IB-IV patients having metastatic LNs, those who underwent chemoradiotherapy had a superior DFS as compared with those who received adjuvant chemotherapy (capecitabine plus cisplatin) only, but still, $<30 \%$ of the enrolled patients had stage III gastric cancer. ${ }^{7}$ Adjuvant chemotherapy and adjuvant chemoradiotherapy are routinely used in clinical practice; however, it is still unclear which subgroups of patients would get an optimal survival benefit from them. The aim of this study was to compare the survival advantage of the two treatments among stage III patients who underwent D2 gastrectomy, and to identify which subgroups of patients can benefit more from adjuvant chemoradiotherapy.

\section{Materials and methods Inclusion and exclusion criteria}

Retrospective data were collected from January 2009 to December 2014. Eligible patients were pathologically diagnosed as stage III disease, as classified according to the eighth edition of Union for International Cancer Control (UICC) and the American Joint Committee on Cancer (AJCC) gastric cancer staging system. The inclusion criteria were as follows: 1) patients aged between 20 and 75 years old, who had undergone radical D2 gastrectomy; 2) those who had histologically confirmed stage III gastric cancer with no clinical or imaging evidence of distant metastasis (M0); 3) patients with postoperative survival $>3$ months. The exclusion criteria were as follows: 1) patients who had undergone neoadjuvant treatment(s); 2) adjuvant radiotherapy alone without any combination of chemotherapy; 3) positive surgical resection margins after surgery; 4) inadequate function of important organs (ie, heart, liver and kidney); and 5) coexisting malignancies, or past history of any other cancer(s) and radio/chemotherapy. The study was approved by the ethics committee of Zhongshan Hospital affiliated to Fudan University and written informed consent was obtained for each patient.

\section{Surgical approaches}

All eligible patients had undergone a radical total/subtotal D2 gastrectomy, comprising resection of all perigastric lymph nodes (LNs) (ie, nodes at the right and left cardia vessels, the short gastric vessels, along the lesser and greater curvature, left and right gastroepiploic vessels, supra- and infra-pyloric regions), including the nodes along the root of the left gastric artery, common hepatic artery, and the splenic artery, around the celiac axis or at the splenic hilum, depending on the location of the primary tumor. Total gastrectomy referred to the en-bloc resection of the stomach and subtotal gastrectomy referred to the removal of the proximal or distal two-third of the stomach. Tumor deposits refer to satellite peritumoral nodules found in the peritumoral adipose tissue of gastric cancer without any histologic evidence of residual LN in the nodule. 


\section{Adjuvant chemotherapy regimens}

All eligible patients received fluoropyrimidine-based regimen every 21 days for 4-6 cycles after recovery from surgery within 8 weeks. Single-drug regimen refers to oral drug of FU, comprising of $40-60 \mathrm{mg}$ of tegafur/ gimeracil/oteracil potassium capsules $\left(\mathrm{S}-1^{\circledR}\right.$, Taiho Pharmaceutical Co. Ltd., Tokyo, Japan; or Tegafur Capsules, Hengrui Medicine Co. Ltd., Jiangsu, P.R. China), twice daily, or $625-825 \mathrm{mg} / \mathrm{m}^{2}$ of oral capecitabine $\left(\right.$ Xeloda $^{\circledR}$, Roche Pharmaceutical Co. Ltd., Basel, Switzerland) twice daily. The doublet regimen refers to the combination of two kinds of chemotherapeutic drugs, comprising of $85-130 \mathrm{mg} / \mathrm{m}^{2}$ of oxaliplatin (Aiheng ${ }^{\circledR}$, Hengrui Medicine Co. Ltd.) or $75-100 \mathrm{mg} / \mathrm{m}^{2}$ of cisplatin (Qilu Pharmaceutical Co. Ltd., Shandong, P.R. China) or $70-85 \mathrm{mg} / \mathrm{m}^{2}$ of docetaxel (Aisu ${ }^{\circledR}$, Hengrui Medicine Co. Ltd.) plus bolus $1,600-2,000 \mathrm{mg} / \mathrm{m}^{2}$ of $\mathrm{FU}$ and 200-400 $\mathrm{mg} / \mathrm{m}^{2}$ of leucovorin (Xudong Hepu Pharmaceutical Co., Ltd., Shanghai, P.R. China) or oral drugs of FU. The triplet regimen refers to three combinations of chemotherapeutic drugs (ie, ECF: epirubicin $\left[50 \mathrm{mg} / \mathrm{m}^{2}\right.$, Haizheng Pharmaceutical Co. Ltd., Zhejiang, P.R. China], platinum plus FU; mDCF: docetaxel $\left[40-75 \mathrm{mg} / \mathrm{m}^{2}\right]$, platinum plus $\mathrm{FU}$ ).

\section{Adjuvant chemoradiotherapy}

Patients were placed in a supine position with thermoplastic immobilization during either intensive modulation radiotherapy (IMRT) or three-dimensional chemoradiotherapy with a 6-MV photon beam. The clinical target volume (CTV) mainly included the anastomotic stoma and regional draining LNs (perigastric, paraoesophageal, paracardial, celiac, splenic, hepatoduodenal or hepatic portal, and pancreaticoduodenal LNs), depending on the location of the primary tumor, and as described previously. ${ }^{8}$ The planning target volume (PTV) was defined and created by the addition of a uniform margin of up to $0.5-1.0 \mathrm{~cm}$ to the CTV, and it was optimized to ensure a dose of $45.0 \mathrm{~Gy}$ to $>95 \%$ of the PTV. High dose of 50.0-54.0 Gy may be used in patients with stage $\mathrm{T} 4 \mathrm{~b}$ disease as a boost to that area, defined by surgical clips. The total radiation dose was administered over 5 weeks, depending on the location of the primary tumor and organs at risk (spinal cord, liver, lung, kidney, bowel, and heart). The maximum dose given to the spinal cord was $<45 \mathrm{~Gy}$, the volume receiving $20 \mathrm{~Gy}$ $\left(\mathrm{V}_{20}\right)$ to the lung was $<35 \%, \mathrm{~V}_{45}$ to the bowel was $<195$ cubic centimeter, $\mathrm{V}_{30}$ to the heart was $<20 \%$, mean dose to the liver was $<25$ Gy and one of $\mathrm{V}_{20}$ to the right or left kidney $<33 \%$. The prescribed concurrent chemotherapy regimens consisted of $200-250 \mathrm{mg} / \mathrm{m}^{2}$ of bolus $\mathrm{FU}$ on days 1-5 per week or $625-825 \mathrm{mg} / \mathrm{m}^{2}$ of oral capecitabine twice on days 1-5 per week for 5 weeks. In the adjuvant chemoradiotherapy group, chemotherapy was given for 3-6 cycles before radiotherapy and continued for $0-3$ cycles after radiotherapy.

\section{Follow-up plan}

After surgery, all patients were followed-up every 3 months in their first preceding year, then every 6 months until 3 years, and yearly thereafter. Follow-up examinations included a history and physical examination, blood chemistry including serum tumor biomarkers (CEA, CA19-9, CA724, CA242), computed tomography scans of the chest, abdomen, and pelvis, as well as upper gastrointestinal endoscopy. Local regional recurrence referred to recurrence at the anatomic stoma, tumor bed, residual stomach, and regional LNs, which were included in the radiation field of the radiotherapy. Peritoneal metastasis was defined as histological evidence of tumor cells in the ascites. Distant metastasis was defined as imaging or pathological evidence of any metastasis to distant LNs outside the radiation field or distant organs (liver, lung, brain, and bone). In the event of disease recurrence or metastasis after the adjuvant strategies, majority of the patients opted for palliative treatments such as radiofrequency treatment, interventional therapy or radiotherapy for liver metastasis, palliative chemotherapy, or traditional Chinese medicine treatment. Adverse effects were assessed using the National Cancer Institute-Common Terminology Criteria for Adverse Effect (version 4.0). In case of appearance of grade III-IV adverse effects, the concurrent chemotherapeutic drugs may be reduced or even discontinued. If the patient still cannot recover from serious adverse effect, radiotherapy will be suspended.

\section{Statistical analysis}

All statistical analyses were performed using the IBM SPSS $^{\circledR}$ software version 22.0 (Chicago, IL, USA). The $\mathrm{LN}$ ratio was calculated as the ratio of the number of metastatic LNs to the total number of LNs examined. The Pearson's Chi-square test (or Fisher's exact test) and the Student's $t$-test were used to identify any significant differences in the investigated baseline characteristics and to detect the significance of 3-, 5-, and 7-year OS and DFS between the adjuvant chemoradiotherapy and chemotherapy 
groups. The Cox's proportional hazards model was used to identify any association of OS with each investigated clinical/demographic factor. After the independent prognostic factors were identified (age, TNM stages, LN ratio, tumor deposits, and total/subtotal gastrectomy), they were adjusted for survival comparison using propensity score-matching (PSM) analysis. Propensity score was calculated by using a logistic regression model with a caliper of width 0.01 and a matching ratio of 1:1. The Kaplan-Meier method was performed to estimate the median OS and to compare the association of OS and DFS with relevant clinicopathological factors between the two treatment groups. All $P$-values were two-sided, and a $P$-value of $<0.05$ was regarded as statistically significant.

\section{Results}

\section{Study population and patient characteristics}

A total of 415 cases with stage III gastric cancer were collected. These consisted of 135 and 280 patients who received adjuvant chemoradiotherapy and chemotherapy, respectively. The mean ages and SD of those receiving adjuvant chemoradiotherapy and chemotherapy were 54.3 \pm 10.7 years and $56.3 \pm 9.6$ years, respectively $(P=0.058)$. The baseline characteristics were comparable except for gastrectomy manner, listed in Table 1.

\section{Adjuvant treatments and adverse events}

The median dose of radiation in patients undergoing adjuvant chemoradiotherapy was $45.0 \mathrm{~Gy}$ (range 45.0-50.0 Gy). About $75.5 \%$ (102/135) of patients in the adjuvant chemoradiotherapy group finished the planned chemotherapy and $88.2 \%(247 / 280)$ of patients in the adjuvant chemotherapy group completed 4-6 cycles of chemotherapy (adjuvant chemoradiotherapy vs adjuvant chemotherapy, mean: 5.1 vs 5.4 cycles; median: 5 vs 5 cycles; range $1-6$ vs $1-6$ cycles). Grade III-IV adverse events were usually presented as leucopoenia/granulocytopoenia, accounting for $16.3 \%$ in the adjuvant chemoradiotherapy group and $10.7 \%$ in the adjuvant chemotherapy group. Grade III-IV hematological adverse events were slightly higher in the adjuvant chemoradiotherapy $(18.5 \%, 25 / 135)$ compared with the adjuvant chemotherapy group $(11.8 \%, 33 / 280) \quad(P=0.089)$. Gastrointestinal disorders (ie, asthenia/anorexia, diarrhea, nausea/vomiting, abdominal pain, and intestinal subocclusion) were also slightly higher in the adjuvant chemoradiotherapy $(28.8 \%, 39 / 135)$ compared with the adjuvant chemotherapy group $(20.7 \%, 58 / 280)(P=0.072)$. Their difference was reduced after PSM analysis. The main reasons for incompletion of the treatments were due to toxicity and disease progression. Also, radiotherapy was suspended for two patients in the adjuvant chemoradiotherapy group due to partial small bowel obstruction and liver dysfunction, but was completed after the patients recovered. No treatmentrelated deaths were observed in this study. Details of the adverse effects are listed in Table 2.

\section{Survival analysis}

The median follow-up time was 41.1 months (range 7.0-104.2 months). There were a total of 250 deaths, including $68(50.4 \%)$ in the adjuvant chemoradiotherapy group and $182(65.0 \%)$ in the adjuvant chemotherapy group at the end of follow-up. The mean time and SE of OS and DFS were higher in the adjuvant chemoradiotherapy group as compared with the adjuvant chemotherapy group (OS, $51.2 \pm 2.5$ vs $42.2 \pm 1.4$, respectively [ $P=0.003$ ]; DFS, $46.5 \pm 2.7$ vs $33.6 \pm 1.5$, respectively $[P=0.002]$ ). The 5- and 7-year OS and DFS rates were significantly higher in the adjuvant chemoradiotherapy group compared with the chemotherapy group (5-year OS: $45.2 \%$ vs $23.6 \%$; 5-year DFS rate: $40.7 \%$ vs $20.0 \%$, respectively, and 7-year OS: $20.0 \%$ vs $6.8 \%$; 7 -year DFS: $18.5 \%$ vs $6.1 \%$, respectively, all $P<0.01$ ) (Figure $1 \mathrm{~A}$ and $\mathrm{B}$ ).

\section{PSM analysis}

Multivariate analysis identified that treatment strategies (adjuvant chemoradiotherapy and adjuvant chemotherapy) (HR, 0.60; 95\% CI: 0.44-0.80), pTNM stage (HR, 1.73; 95\% CI: $1.26-2.38$ ), LN ratio $\geq 50 \%$ (HR, 1.81; 95\% CI: 1.33-2.48), tumor deposits (HR, 1.42; 95\% CI: 1.04-1.95), and gastrectomy manner (HR, $1.21 ; 95 \% \mathrm{CI}: 1.05-1.40$ ) were independent prognostic factors for OS (Table 3). When the PSM analysis was adjusting by these factors, 135 patients in each group were matched. The patients' baseline characteristics were consistent with each other, listed in Table 1. The results demonstrated that patients who had adjuvant chemoradiotherapy had superior OS than those who had adjuvant chemotherapy (median OS, 51.2 vs 39.3 months; HR, $0.60,95 \%$ CI: $0.44-0.83, P<0.01 ; 5$ - and 7-year OS: $45.2 \%$ vs $19.3 \%$ and $20.0 \%$ vs $6.7 \%$, respectively). By PSM analysis, the long-term OS and DFS were also superior in the adjuvant chemoradiotherapy group compared with adjuvant chemotherapy (5- and 7-year DFS: $40.7 \%$ vs $16.3 \%$ and $18.5 \%$ vs $5.2 \%$, respectively, all $P<0.01$ ) (Figure $1 \mathrm{C}$ and $\mathrm{D}$ ). 
Table I The baseline characteristics of patients with locally advanced gastric cancer after D2 gastrectomy before and after PSM

\begin{tabular}{|c|c|c|c|c|c|}
\hline \multirow[t]{2}{*}{ Subgroup } & \multirow{2}{*}{$\begin{array}{l}\text { Chemoradiotherapy } \\
\mathrm{N}=135, \mathrm{n}(\%)\end{array}$} & \multirow{2}{*}{$\begin{array}{l}\text { Chemotherapy } \\
\text { Before PSM } \\
\mathrm{N}=\mathbf{2 8 0}, \mathrm{n}(\%)\end{array}$} & \multirow[t]{2}{*}{$P$-value } & \multirow{2}{*}{$\begin{array}{l}\text { Chemotherapy } \\
\text { After PSM } \\
N=135, n \text { (\%) }\end{array}$} & \multirow[t]{2}{*}{$P$-value } \\
\hline & & & & & \\
\hline \multicolumn{6}{|l|}{ Age (years) } \\
\hline Mean \pm SD & $54.3 \pm 10.7$ & $56.3 \pm 9.6$ & 0.058 & $54.7 \pm 10.2$ & 0.740 \\
\hline \multicolumn{6}{|l|}{ Sex } \\
\hline Male & 97 (7I.9) & $195(69.6)$ & 0.644 & $89(65.9)$ & 0.293 \\
\hline Female & $38(28.1)$ & $85(30.4)$ & & $46(34.1)$ & \\
\hline \multicolumn{6}{|c|}{ AJCC/UICC pTNM classification } \\
\hline IIIA & $39(28.9)$ & $110(39.3)$ & 0.093 & $38(28.1)$ & 0.717 \\
\hline IIIB & $56(4 \mid .5)$ & $92(32.9)$ & & $51(37.8)$ & \\
\hline IIIC & $40(29.6)$ & $78(27.8)$ & & $46(34.1)$ & \\
\hline \multicolumn{6}{|c|}{ AJCC/UICC pT classification } \\
\hline $\mathrm{T} 2$ & $8(5.9)$ & $10(3.6)$ & 0.415 & $4(3.0)$ & 0.305 \\
\hline T3 & $21(15.6)$ & $53(18.9)$ & & $28(20.7)$ & \\
\hline $\mathrm{T} 4$ & $106(78.5)$ & $217(77.5)$ & & $103(76.3)$ & \\
\hline \multicolumn{6}{|c|}{ AJCC/UICC pN classification } \\
\hline No 2 & $42(31.1)$ & II 5 (4I.I) & 0.121 & $4 \mathrm{I}(30.4)$ & 0.081 \\
\hline N3a & $61(45.2)$ & $102(36.4)$ & & $60(44.4)$ & \\
\hline N3b & $32(23.7)$ & $63(22.5)$ & & $34(25.2)$ & \\
\hline \multicolumn{6}{|l|}{$\mathrm{LN}$ ratio } \\
\hline$<50 \%$ & $91(67.4)$ & $205(73.2)$ & 0.220 & $99(73.3)$ & 0.286 \\
\hline$\geq 50 \%$ & $44(32.6)$ & $75(26.8)$ & & $36(26.7)$ & \\
\hline \multicolumn{6}{|l|}{ Tumor deposits } \\
\hline Absent & $110(81.5)$ & $224(80.0)$ & 0.721 & 105 (77.8) & 0.450 \\
\hline Present & $25(18.5)$ & $56(20.0)$ & & $30(22.2)$ & \\
\hline \multicolumn{6}{|l|}{ Histological type } \\
\hline Adenocarcinoma & I23 (9I.I) & $262(93.6)$ & 0.365 & $127(94.1)$ & 0.353 \\
\hline SRC or mucinous & $12(8.9)$ & $18(6.4)$ & & $8(5.9)$ & \\
\hline \multicolumn{6}{|l|}{ Tumor location } \\
\hline Antrum & $62(45.9)$ & $103(36.8)$ & 0.192 & $53(39.3)$ & 0.536 \\
\hline Cardia & $23(17.0)$ & $60(2 \mathrm{I} .4)$ & & $25(18.5)$ & \\
\hline Body & $50(37.0)$ & $117(4 \mid .8)$ & & $57(42.2)$ & \\
\hline \multicolumn{6}{|c|}{ Chemotherapy regimens* } \\
\hline Single & II (8.I) & $22(7.9)$ & 0.316 & $6(4.4)$ & 0.134 \\
\hline Doublet & $102(75.6)$ & $227(81.0)$ & & $115(85.2)$ & \\
\hline Triplet & $22(16.3)$ & $31(11.1)$ & & $14(10.4)$ & \\
\hline Gastrectomy & & & & & \\
\hline Subtotal & $107(79.3)$ & $196(70.0)$ & 0.047 & $108(80.0)$ & 0.880 \\
\hline Total & $28(20.7)$ & $84(30.0)$ & & $27(20.0)$ & \\
\hline
\end{tabular}

Note: *Single regimens refers to oral drug of fluorouracil. The doublet regimen included oxaliplatin or cisplatin or docetaxel plus bolus fluorouracil and leucovorin or oral $\mathrm{S}$-1/capecitabine. The triplet regimen comprised of the above-mentioned doublet regimen plus docetaxel or epirubicin.

Abbreviations: $\mathrm{N}$, total number of patients in the corresponding treatment group; $n$, number of patients in the corresponding group; PSM, Propensity score-matched; SRC, signet ring cell carcinoma. 
Table 2 Grade III-IV toxicities occurring in the process of the adjuvant chemoradiotherapy and adjuvant chemotherapy

\begin{tabular}{|c|c|c|c|c|c|}
\hline \multirow[t]{2}{*}{ Grade III-IV toxicities } & \multicolumn{2}{|c|}{ Chemoradiotherapy } & \multicolumn{2}{|c|}{ Chemotherapy } & \multirow[t]{2}{*}{$P$-value } \\
\hline & $N=135, n(\%)$ & $\begin{array}{l}\text { Before PSM } \\
N=280 \text {, n (\%) }\end{array}$ & $P$-value & $\begin{array}{l}\text { After PSM } \\
N=135, \text { n (\%) }\end{array}$ & \\
\hline Leucopoenia/granulocytopenia & $22(16.3)$ & $30(10.7)$ & 0.108 & $18(13.3)$ & 0.493 \\
\hline Anemia & $2(1.5)$ & I $(0.4)$ & 0.205 & I (0.7) & 0.562 \\
\hline Thrombocytopenia & $\mathrm{I}(0.7)$ & $2(0.7)$ & 0.976 & $2(1.5)$ & 0.562 \\
\hline Asthenia/anorexia & $20(14.8)$ & $32(11.4)$ & 0.076 & $15(11.1)$ & 0.365 \\
\hline Diarrhea & $2(1.5)$ & $2(0.7)$ & 0.454 & $\mathrm{I}(0.7)$ & 0.562 \\
\hline Nausea/vomiting & $12(8.9)$ & $21(7.5)$ & 0.624 & $10(7.4)$ & 0.615 \\
\hline Abdominal pain & $4(3.0)$ & $2(0.7)$ & 0.072 & $\mathrm{I}(0.7)$ & 0.176 \\
\hline Intestinal subocclusion & $\mathrm{I}(0.7)$ & $\mathrm{I}(0.4)$ & 0.597 & $0(0.0)$ & 0.316 \\
\hline Liver dysfunction & $\mathrm{I}(0.7)$ & $2(0.7)$ & 0.976 & $0(0.0)$ & 0.316 \\
\hline
\end{tabular}

Abbreviations: $\mathrm{N}$, total number of patients in the corresponding treatment group; $\mathrm{n}$, number of patients in the corresponding group.

A

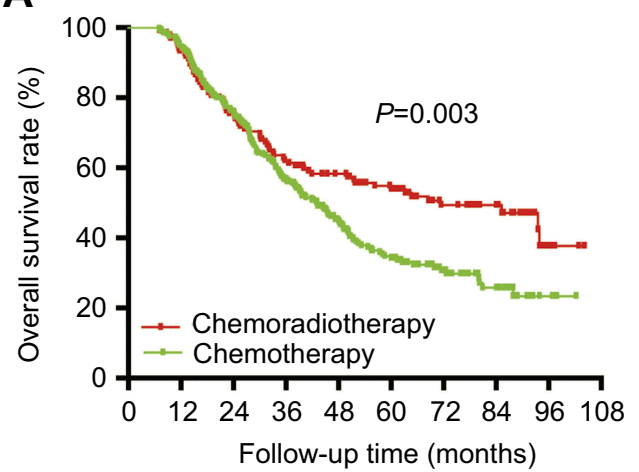

No.at risk

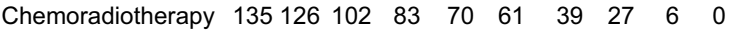

$\begin{array}{lllllllllll}\text { Chemotherapy } & 280 & 266 & 214 & 154 & 100 & 66 & 37 & 19 & 4 & 0\end{array}$

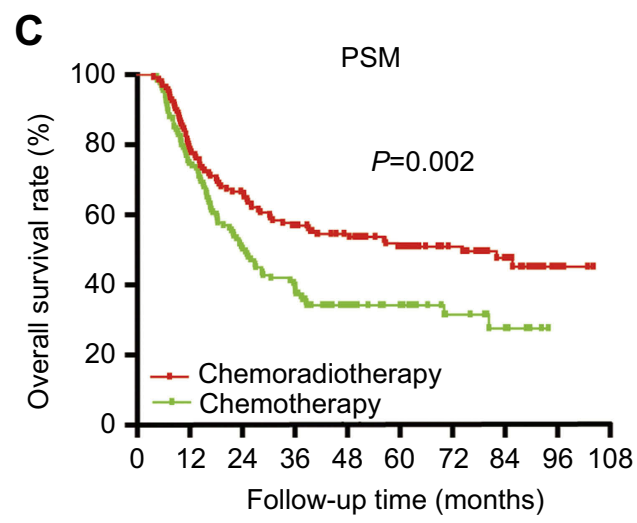

No.at risk

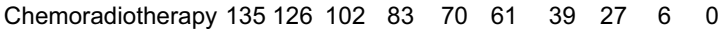

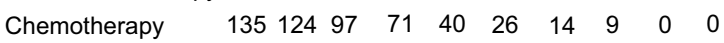

B

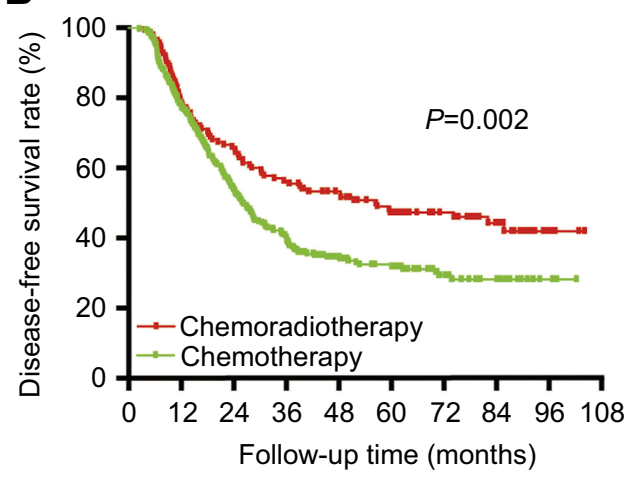

No.at risk

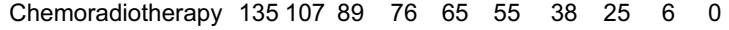

$\begin{array}{lllllllllll}\text { Chemotherapy } & 280 & 219 & 150 & 110 & 70 & 55 & 31 & 17 & 4 & 0\end{array}$

Figure I Survival curves showing the overall survival (OS) and disease-free survival (DFS) in the entire and propensity score-matched (PSM) cohorts. Patients in the adjuvant chemotherapy group had a significant OS (A) and DFS (B) advantage than the adjuvant chemotherapy in the entire cohort. These survival advantages were enhanced in the PSM cohort (C and D). 
Table 3 Prognostic factors were identified using the Cox proportional hazards model

\begin{tabular}{|c|c|c|c|c|c|}
\hline \multirow[t]{2}{*}{ Variable } & \multicolumn{3}{|c|}{ Univariate } & \multicolumn{2}{|c|}{ Multivariate } \\
\hline & HR & $P$-value & HR & $95.0 \% \mathrm{Cl}$ & $P$-value \\
\hline Chemoradiotherapy vs chemotherapy* & 0.60 & 0.003 & 0.60 & $0.44-0.80$ & 0.001 \\
\hline Age stratification ( $<40$ years, $40-60$ vs $>60$ years) & 0.83 & 0.082 & 0.74 & $0.59-0.93$ & 0.069 \\
\hline Sex (male vs female) & 1.20 & 0.170 & 0.99 & $0.75-1.30$ & 0.914 \\
\hline PTNM stage (IIIA, IIIB vs IIIC) & 1.61 & $<0.001$ & 1.73 & $1.26-2.38$ & $<0.001$ \\
\hline AJCC/UICC pT classification ( $\leq \mathrm{T} 2, \mathrm{~T} 3$ vs $\mathrm{T} 4)$ & 1.41 & 0.012 & 1.17 & $0.88-1.57$ & 0.098 \\
\hline AJCC/UICC pN classification ( $\leq \mathrm{NI}, \mathrm{N} 2$ vs N3) & 1.26 & $<0.001$ & 0.83 & $0.67-1.02$ & 0.067 \\
\hline LN ratio $\geq 50 \%$ vs $L N$ ratio $\leq 50 \%$ & 2.30 & $<0.001$ & 1.81 & $1.33-2.48$ & $<0.001$ \\
\hline Tumor deposits (with vs without) & 1.52 & 0.006 & 1.42 & $1.04-1.95$ & 0.028 \\
\hline Histological type (adenocarcinoma vs others ${ }^{\#}$ ) & 1.14 & 0.0570 & 1.04 & $0.65-1.68$ & 0.873 \\
\hline Tumor location (antrum, cardia vs body) & 1.02 & 0.773 & 0.94 & $0.8 \mathrm{I}-1.09$ & 0.398 \\
\hline Chemotherapy regimens (single, doublet vs triplet) & 0.86 & 0.291 & 0.89 & $0.66-1.19$ & 0.437 \\
\hline Total vs subtotal gastrectomy & 1.29 & $<0.001$ & 1.21 & $1.05-1.40$ & 0.007 \\
\hline
\end{tabular}

Notes: *refers to adjuvant therapy; ${ }^{\#}$ refers to signet ring cell carcinoma and mucinous adenocarcinoma.

Abbreviations: $L N$, lymph node; $p$, pathological stage.

\section{Recurrence patterns}

The key relapse sites were classified into three categories: locoregional recurrence (refers to relapse in radiation field of CTV in patients receiving adjuvant chemoradiotherapy), abdominal or pelvic cavity peritoneal tumor seeding, and distant metastases and were compared between the two treatment groups. We observed that total recurrence was significantly more frequent in the adjuvant chemotherapy group compared with the adjuvant chemoradiotherapy group (70.4\% vs $53.3 \%$, respectively $[P<0.001]$ ), especially in locoregional recurrence $(21.1 \%$ vs $7.4 \%$, respectively $[P<0.001])$. Also, we observed that in both groups, patients with N3 stage disease had a higher risk of total recurrence than those with N0-2 stage disease (adjuvant chemoradiotherapy, $58.1 \%$ vs $38.1 \%$, respectively [ $P=0.032]$; adjuvant chemotherapy, $74.5 \%$ vs $62.6 \%$, respectively $[P=0.033])$. The incidence of peritoneal seeding and distant metastasis was not significantly different between the two groups (Table 4).

\section{Subgroup analyses}

Patients had significant OS rate in terms of TNM stages (pIIIA, pIIIB, and pIIIB) (Figure 2A). For subset analysis, patients with stage pIIIA and pIIIB LAGC were more likely to benefit from adjuvant chemoradiotherapy versus adjuvant chemotherapy (5-year OS rate, pIIIA: $61.5 \%$ vs $34.5 \%$ [ $P=0.030]$; pIIIB: $46.4 \%$ vs $26.1 \%[P=0.035])$ (Figure $2 \mathrm{~B}$ and $\mathrm{C}$ ), while patients with stage pIIIC did not benefit from adjuvant chemoradiotherapy $(P=0.180)$, but a survival advantage seems to appear 3 years later in the long-time OS, and 5- and 7-year OS rates were significantly higher in adjuvant chemoradiotherapy group than the other group (5-year OS: $27.5 \%$ vs $5.1 \%$, respectively [ $P=0.001]$; 7 -year OS: $17.5 \%$ vs $1.3 \%$; respectively, both $[P=0.002]$ ) (Figure 2D).

The results of the subset analysis including HRs and 95\% CIs for OS are illustrated as Forest plots in Figure 3, identifying that adjuvant chemoradiotherapy was an important

Table 4 Patients in the adjuvant chemoradiotherapy group had a lower locoregional relapse than the adjuvant chemotherapy

\begin{tabular}{|c|c|c|c|c|c|c|c|}
\hline \multirow[t]{3}{*}{ Failure patterns } & \multicolumn{2}{|c|}{ Chemotherapy, n (\%) } & \multirow[b]{2}{*}{ Total } & \multicolumn{3}{|c|}{ Chemoradiotherapy, n (\%) } & \multirow[t]{3}{*}{$P$-value } \\
\hline & No-2 & N3 & & No-2 & N3 & Total & \\
\hline & $n=1 \mid 5$ & $n=165$ & $\mathbf{N}=\mathbf{2 8 0}$ & $n=42$ & $n=93$ & $N=135$ & \\
\hline No relapse & $43(37.4)$ & $42(25.5)$ & $83(29.6)$ & $26(61.9)$ & 39 (4I.9) & $63(46.7)$ & $<0.001$ \\
\hline Relapse* & $72(62.6)$ & $123(74.5)$ & $197(70.4)$ & $16(38.1)$ & $54(58.1)$ & $72(53.3)$ & $<0.001$ \\
\hline Locoregional & $20(17.4)$ & $39(23.6)$ & $59(21.1)$ & $2(4.8)$ & $8(8.6)$ & $10(7.4)$ & $<0.001$ \\
\hline Peritoneum & $30(26.1)$ & $55(33.3)$ & $79(28.2)$ & $10(23.8)$ & $25(26.9)$ & $35(24.4)$ & 0.625 \\
\hline Distant & $28(24.3)$ & $43(26.1)$ & $59(2 I . I)$ & $6(14.3)$ & $23(24.7)$ & $29(21.5)$ & 0.924 \\
\hline
\end{tabular}

Note: *Some patients have more than one site of relapse. P-value compared failure patterns between adjuvant chemoradiotherapy and chemotherapy. 


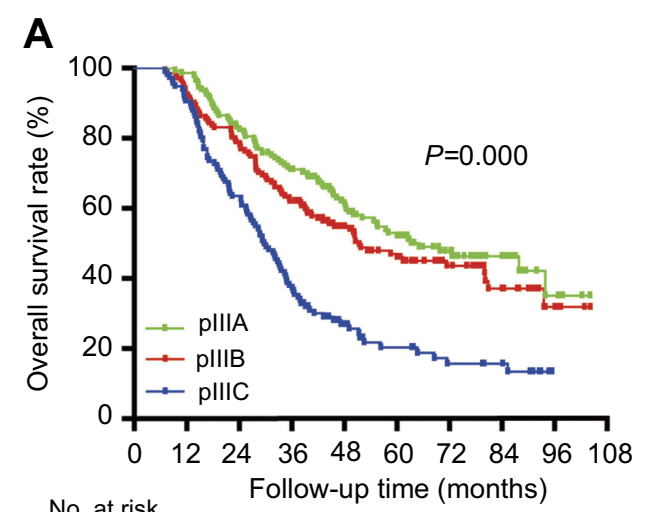

No. at risk

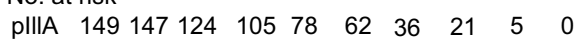

plIIB $148 \begin{array}{lllllllll}138 & 117 & 90 & 68 & 50 & 30 & 17 & 5 & 0\end{array}$

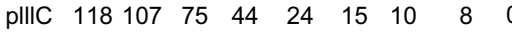

C

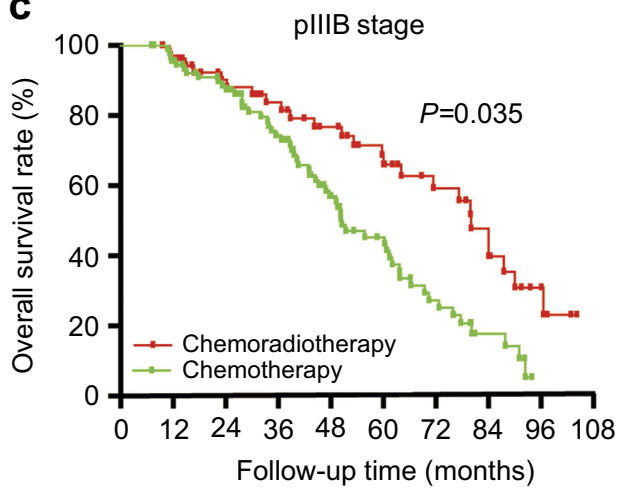

No. at risk

Chemoradiotherapy $\begin{array}{llllllllll}56 & 52 & 43 & 37 & 31 & 26 & 17 & 12 & 5 & 0\end{array}$

$\begin{array}{llllllllll}\text { Chemotherapy } & 92 & 86 & 74 & 53 & 37 & 24 & 13 & 5 & 0\end{array}$
B

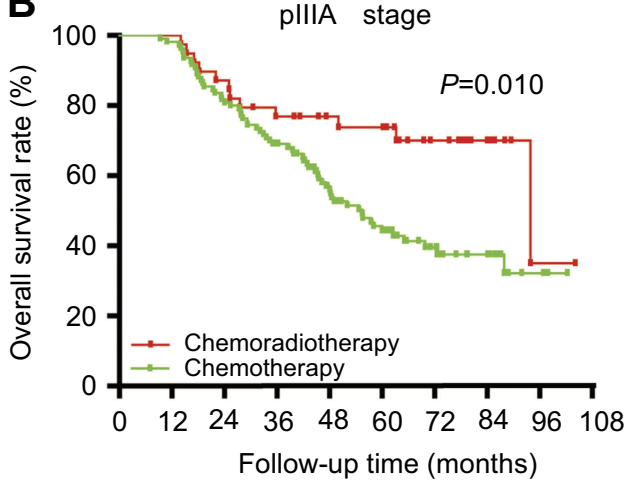

No. at risk

Chemoradiotherapy $\begin{array}{llllllllll}39 & 39 & 34 & 29 & 25 & 24 & 14 & 8 & 1 & 0\end{array}$

$\begin{array}{lllllllllll}\text { Chemotherapy } & 110 & 108 & 90 & 76 & 53 & 38 & 22 & 13 & 4 & 0\end{array}$

D pllIC stage

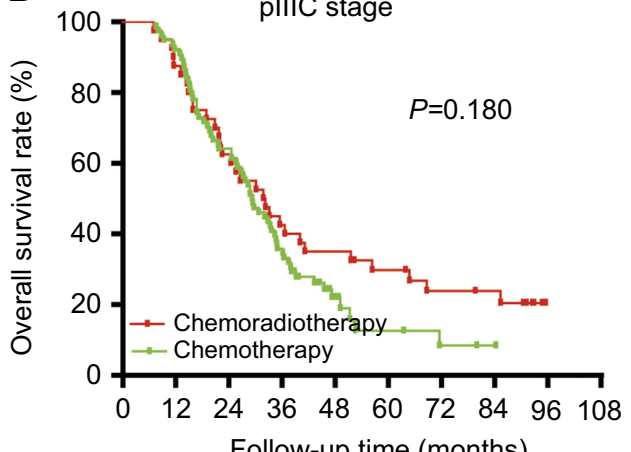

No. at risk

Chemoradiotherapy $\begin{array}{lllllllll}40 & 35 & 25 & 17 & 14 & 11 & 8 & 7 & 0\end{array}$

$\begin{array}{llllllllll}\text { Chemotherapy } & 78 & 72 & 50 & 27 & 10 & 4 & 2 & 1 & 0\end{array}$

Figure 2 Survival curves showing the association of overall survival (OS) with different TNM stage in the entire cohorts. Significant survival difference was observed among patients with pIIIA-pIIIC (A). There were significant survival advantages in patients with pIIIA (B) and pllIB (C) stage disease, but not in those with pllIC (D) stage disease. The 3-, 5-, and 7-year OS rates were estimated and compared between the two treatment groups by Pearson's Chi-square test.

beneficial factor associated with survival advantage in most subsets. For example, patients with pIIIA and pIIIB stage disease, having $\mathrm{LN}$ ratio $<50 \%$, without perigastric tumor deposits, and those undergoing total gastrectomy, might get OS benefits from adjuvant chemoradiotherapy (all $P<0.05$ ). Moreover, patients with signet ring cell carcinoma or mucinous adenocarcinoma appeared to have better survival from adjuvant chemoradiotherapy than for patients with common adenocarcinoma (HR, 0.12, 95\% CI: 0.04-0.44, $P=0.001$ ). Patients with tumors located at the antrum and those undergoing subtotal gastrectomy had a better prognosis and may benefit from adjuvant chemoradiotherapy as compared with chemotherapy. In contrast, patients with tumors located at the gastric cardia or body and those usually undergoing total gastrectomy had a poorer prognosis. However, patients with earlier stage LAGC, such as pT2, or those treated with a single chemotherapeutic drug did not get superior survival from adjuvant chemoradiotherapy (Figure 3).
The median number of resected LNs was 31.7 and 29.8 for the adjuvant chemotherapy and adjuvant chemoradiotherapy groups, respectively, and the median number of metastatic LNs was 10.5 and 10.8 for the two groups, respectively $(P>0.05)$. In the entire cohort, patients with $\mathrm{LN}$ ratio $<50 \%$ had superior OS as compared with patients with $\mathrm{LN}$ ratio $\geq 50 \%$ (Figure $4 \mathrm{~A}$ ). Stratified by four-tier LN ratio classification $(0 \%, 1 \%-9 \%, 10 \%-25 \%$, and $>25 \%)$, our primary results showed that patients with an LN ratio $\geq 25 \%$ may have prolonged OS from adjuvant chemoradiotherapy versus adjuvant chemotherapy (log-rank, $P=0.004$, 5-year OS: $37.8 \%$ vs $16.7 \%$, respectively $[P<0.001]$; and 7 -year OS: $17.8 \%$ vs $3.1 \%$, respectively $[P<0.001])$. When separately stratified as two different classifications, the LN ratio $<50 \%$ and $\geq 50 \%$ cohort, our results showed that adjuvant chemoradiotherapy was beneficial only for those with LN ratio $<50 \%$ (Figure $4 \mathrm{~B}$ ), whereas patients with an LN ratio $\geq 50 \%$ did not 


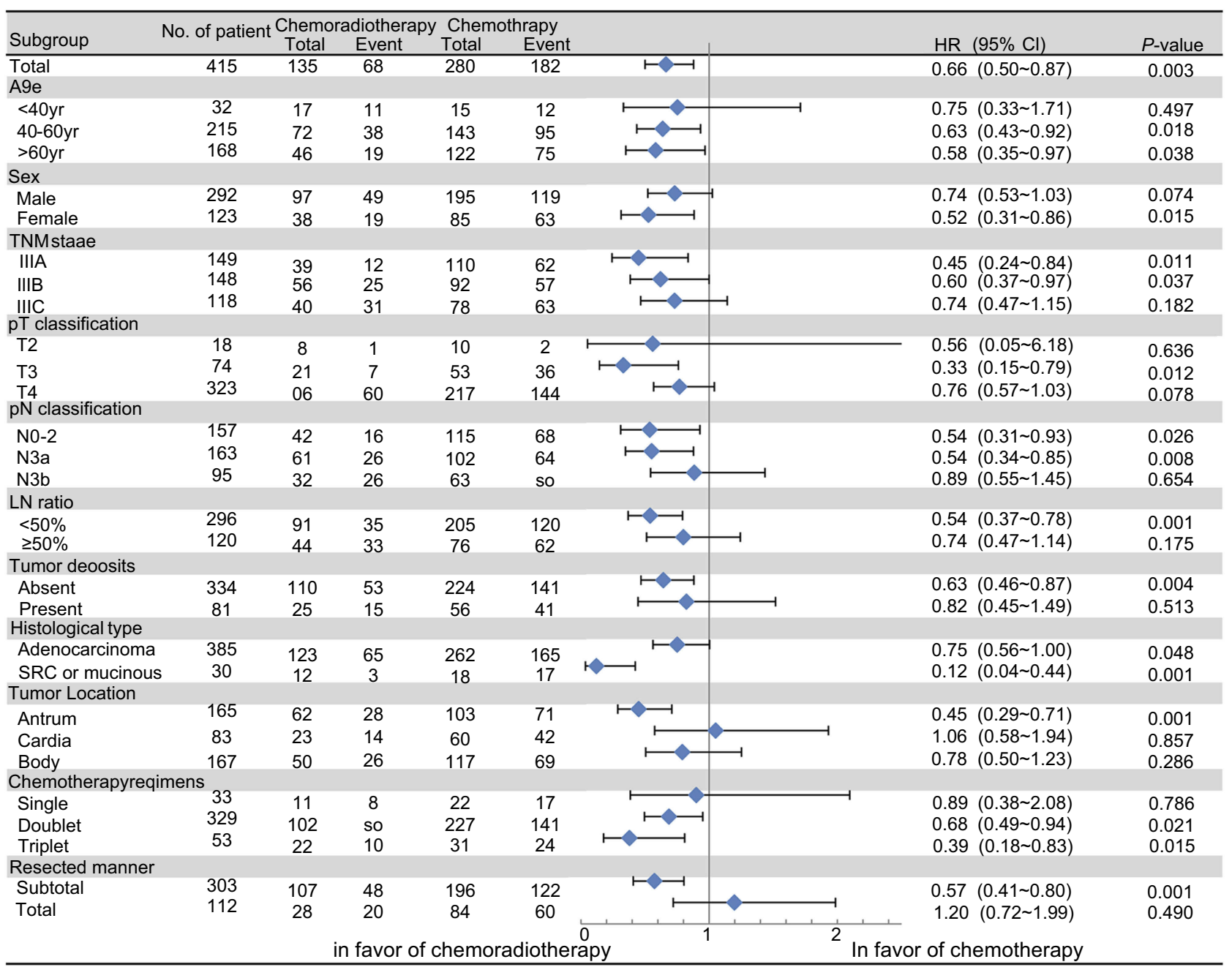

Figure 3 Forest plot showing the association of each clinical feature with overall survival (OS). The OS of all enrolled patients was compared between the groups receiving adjuvant chemoradiotherapy and chemotherapy by univariate analysis. For each factor, a HR, $95 \% \mathrm{Cls}$, and a proportional hazard regression $P$-value was calculated using the Cox proportional hazards model. $H R<I$ favors adjuvant chemoradiotherapy; HR>I favors adjuvant chemotherapy.

Abbreviation: SRC, signet ring cell carcinoma.

experience any survival benefit. However, the 5- and 7-year OS rates were still improved by adjuvant chemoradiotherapy versus chemotherapy group of patients with LN ratios $\geq 50$ (5-year OS: $27.3 \%$ vs $8.2 \%$, 7-year OS: $15.9 \%$ vs $1.4 \%$, both $P<0.01$ ) (Figure $4 \mathrm{C}$ )).

Perigastric tumor deposit was an important prognostic factor for OS (HR, 1.42; 95\% CI: 1.04-1.95). The rate of detection of tumor deposits was $19.3 \%$ (80/415). Patients with tumor deposits had poorer OS compared with those without them (median survival: 32.0 vs 50.3 months; $P=0.005$ ) (Figure 4D). Patients without tumor deposits had a significantly superior OS when they received adjuvant chemoradiotherapy as compared with chemotherapy, especially in the long term (5-year OS rate: $47.3 \%$ vs $25.0 \%, P<0.001$ ) (Figure 4E). However, no significant difference between the two treatment groups was observed for patients with perigastric tumor deposits (Figure 4F).

Chemotherapy regimens were chosen according to patients' tumor characteristics and performance status. In Figure 3, doublet and triplet therapy were found to be in favor of adjuvant chemoradiotherapy as compared with adjuvant chemotherapy $(P=0.021, P=0.015)$. The triplet regimen demonstrated no OS benefit compared with doublet regimen in either the adjuvant chemotherapy or adjuvant chemotherapy by Kaplan-Meier analysis (log-rank, $P=0.510, \quad P=0.176$, respectively). Subgroup analysis showed patients younger than 40 years did not have a superior survival from adjuvant chemoradiotherapy versus adjuvant chemotherapy (5-year OS rates, $25.0 \%$ vs $31.1 \% \quad[P=0.474] ; \quad$ HR, $0.75, \quad 95 \% \quad$ CI: $0.33-1.71$ 

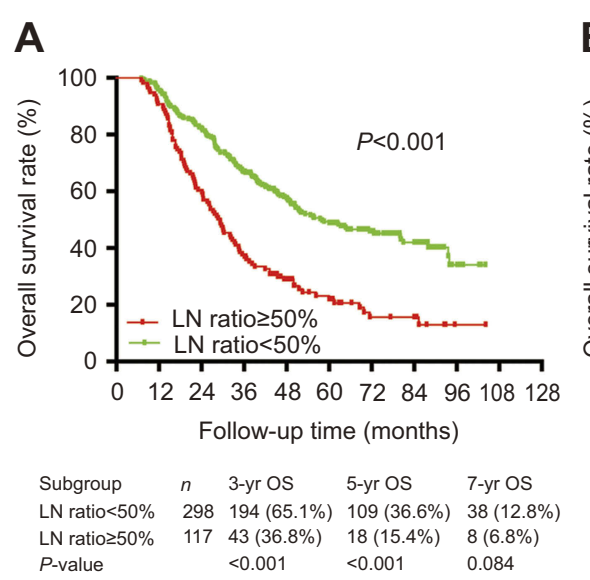

D

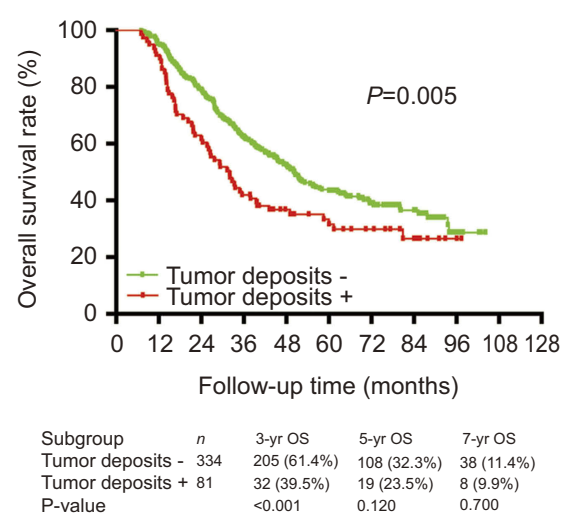

B
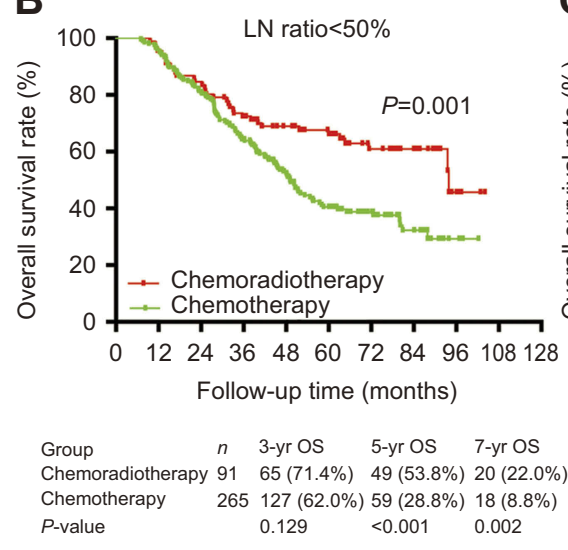

E

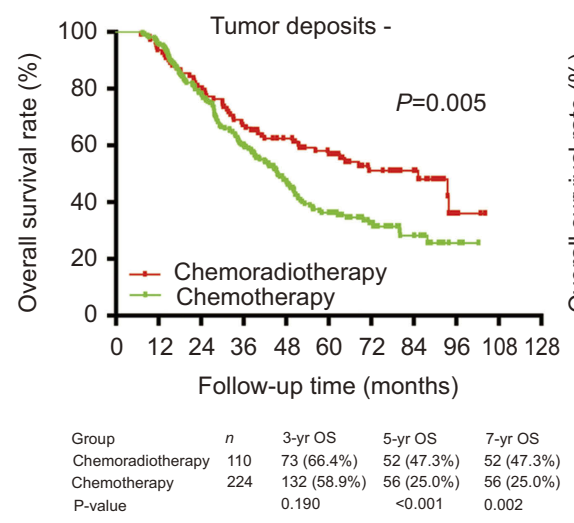

C

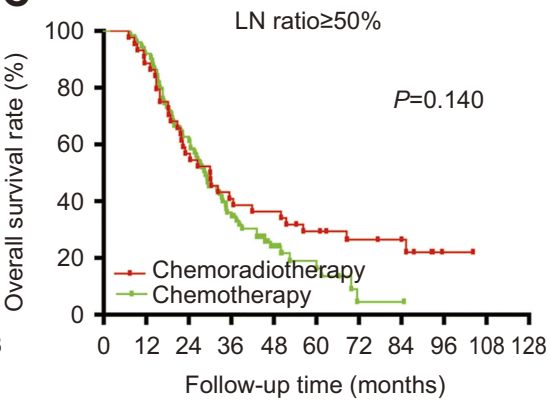

Group $\quad n \quad 3$-yr OS 5-yr OS 7-yr OS Chemoradiotherapy $44 \quad 18(40.9 \%) \quad 12(27.3 \%) 7(15.9 \%)$ Chemotherapy $\quad 73 \quad 25(34.2 \%) \quad 6(8.2 \%) \quad 1(1.4 \%)$ $\begin{array}{llll}P \text {-value } & 0.469 & 0.006 & 0.004\end{array}$

$\mathbf{F}$

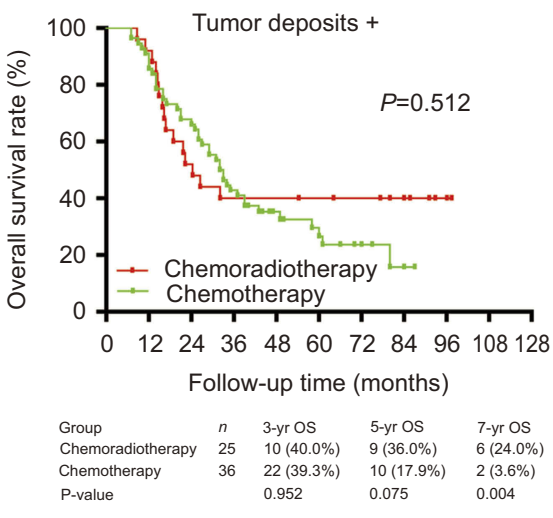

Figure 4 Survival curves showing the association of overall survival (OS) and with lymph node (LN) ratio and tumor deposits. Patients with $L N$ ratio $<50 \%$ had an advantage in OS (A). Better OS (B) survival was shown with adjuvant chemoradiotherapy versus chemotherapy in terms of LN ratio<50\%, but no OS benefit was identifiable in (C) in terms of $L N$ ratio $\geq 50 \%$. OS advantage was observed in patients without perigastric tumor deposits versus patients with them (D). Patients without tumor deposits in the adjuvant chemoradiotherapy group had a superior OS compared with the adjuvant chemotherapy group (E), and those with tumor deposits had no improvement in OS (F). Abbreviations: -, negative; +, positive.

$[P=0.497])$. Patients with antral tumors, or those undergoing subtotal gastrectomy, had a better prognosis and may benefit more from adjuvant chemoradiotherapy. There was no survival advantage from adjuvant chemoradiotherapy in patients with tumors located in the gastric cardia or body, or in those undergoing total gasterctomy. Our limited available retrospective data found no significant differences between the adjuvant chemoradiotherapy and adjuvant chemotherapy groups in terms of Lauren classification status, degree of differentiation, and venous or neural invasion.

\section{Discussion}

This study was designed to compare the treatment efficacies of adjuvant chemoradiotherapy against that of adjuvant chemotherapy, in terms of OS and DFS, in patients with stage III disease who had underwent D2 gastrectomy and to identify which subgroups of patients demonstrated better survival advantage to the superior treatment. Our results demonstrated that patients who treated with adjuvant chemoradiotherapy had superior OS and DFS. Further, subgroup analysis identified that patients with pIIIA and pIIIB stage disease, LN ratio $<50 \%$, antral tumors, and those who had subtotal gastrectomy can have prolonged survival from adjuvant chemoradiotherapy, while no significant difference in survival between the two treatment strategies for patients with tumor deposits, $\mathrm{pN} 3 \mathrm{~b}$ classification, or pIIIC stage disease was observed.

Previous studies showed a 5-year OS advantage of adjuvant chemoradiotherapy versus adjuvant chemotherapy (5-year OS: $40.0-46.7 \%$ vs $20.9-41.0 \%)$. ${ }^{6}$ Our results were in agreement with previous results which demonstrated that adjuvant chemoradiotherapy improved OS compared with adjuvant chemotherapy among patients with stage III LAGC (median OS, 51.2 vs 42.2 months, 
$P<0.01)$. This survival advantage was expanded by PSM analysis. The OS of our patients appears to be longer than the previous studies. Not only did our patients receive fluoropyrimidine-based chemoradiotherapy (5-FU + radiotherapy), but they also received the addition radiotherapy plus adjuvant doublet or triplet chemotherapy. Our subgroup analysis demonstrated that patients had prolonged OS from adjuvant chemoradiotherapy over adjuvant chemotherapy in both subgroups of patients who received either doublet or triplet regimen. Although the chemotherapy scheme was quite heterogeneous, most of them received fluoropyrimidine-based chemotherapy, but the baseline of patients received triplet or doublet regimen, which was consistent with each other in the two groups. However, some future studies are still needed to improve the consistency of the chemotherapy regimens for comparison.

Selecting the group of patients who are most likely to benefit from adjuvant therapy has been a debatable topic. Several randomized clinical trials found no difference in survival between patients receiving adjuvant chemoradiotherapy and chemotherapy, but these studies included a large number of stage II patients, rather than stage III, who were supposed to have a better survival with surgery alone. ${ }^{6,9,10}$ A meta-analysis of 13 randomized trials, consisting of patients with LAGC, showed that adjuvant chemoradiotherapy resulted in approximately $20 \%$ improvements in both OS and DFS, but data did not identify any specific subgroup of patients who did not benefit from adjuvant chemoradiotherapy. ${ }^{11}$ Another metaanalysis found a significant increase in 5-year DFS for adjuvant chemoradiotherapy over chemotherapy (RR, $0.89,95 \%$ CI: $0.81-0.98$ ), but not in OS (RR, $1.05,95 \%$ CI: $0.88-1.25) .^{12}$ In this study, we only included patients with pathological stage III LAGC, and our results showed that patients with pIIIA and pIIIB stage disease had greater benefit from adjuvant chemoradiotherapy as compared with adjuvant chemotherapy.

The randomized Phase III ARTIST trial highlighted the importance of evaluating LN status. However, patients were divided into LN-positive or -negative groups, irrespective of specific $\mathrm{N}$ stage. ${ }^{13}$ One study found that adjuvant chemoradiotherapy for LAGC after D2 gastrectomy was more beneficial in cancers with $\mathrm{LN}$ ratio $>25 \%$ according to the four-tier $\mathrm{LN}$ ratio classification $(0 \%$, $1-9 \%, \quad 10-25 \%$, and $>25 \%){ }^{7}$ Similar results were observed in our preliminary analysis, when we further explored and stratified the LN ratio as separate subgroups. Our data showed that patients with an LN ratio $\geq 50 \%$ did not demonstrate an OS benefit from adjuvant chemoradiotherapy. Further, an Asia study found that the patients after D2 gastrectomy with N3 stage disease had no survival benefit from adjuvant chemoradiotherapy versus adjuvant chemotherapy. ${ }^{14}$ Our subset analysis further demonstrated that adjuvant chemoradiotherapy could prolong the OS of patients with $\mathrm{pN} 0-\mathrm{pN} 3 \mathrm{a}$ but not $\mathrm{pN} 3 \mathrm{~b}$ stage disease. This could be because pN3b patients have a poorer prognosis with a higher risk of distant metastases, which cannot benefit from locoregional treatment; thus, emphasis should be placed on optimizing a more personalized treatment for them.

The CRITICS study showed that adjuvant chemoradiotherapy did not improve OS compared with adjuvant chemotherapy in patients treated with adequate preoperative chemotherapy and surgery. ${ }^{15}$ However, only $60 \%$ of randomized patients were included in the postoperative analysis; this is not feasible for a direct comparison of the two treatments. Furthermore, only $50 \%$ of patients completed the entire study, which may contribute to a population bias. In this study, we excluded the patients who had neoadjuvant treatment since their status may affect the pathological TNM stage of the tumor.

Tumor deposits refer to all perigastric tumor nodules without evidence of residual $\mathrm{LN}$ tissue as regional LN metastases and is distinguished from peritoneal or mesenteric tumor seeding. ${ }^{16}$ Gastric cancer patients with tumor deposits had a higher likelihood of developing recurrence and cancer-related death. ${ }^{17} \mathrm{~A}$ previous study observed perigastric tumor deposits to be present in $23.9 \%$ (156/ 653) of their investigated patients, associated with synchronous distant metastasis. ${ }^{18}$ Another study consisting of 2,998 gastric cancer patients found tumor deposits were detected in $17.8 \%$ of their patients. The prognosis of pT1-4a patients with tumor deposits was similar to that of pT4a patients without tumor deposits, suggesting that this might be appropriately treated as a form of serosal invasion. ${ }^{19}$ Similar to this study, our current study showed tumor deposits were detected in 80 of 415 patients (19.3\%). The survival of patients with tumor deposits was significantly poorer than that of those without them, with no significant difference in relation to adjuvant chemoradiotherapy or adjuvant chemotherapy, which suggests that patients with tumor deposits may demonstrate a more aggressive clinical course, and may thus not benefit from adjuvant chemoradiotherapy.

Subset analysis revealed that patients with most pathological types of cancer have superior survival from 
adjuvant chemoradiotherapy, including adenocarcinoma, but more notably signet ring cell carcinoma and mucinous adenocarcinomas. Despite the possibility of some bias due to the limited number of patients $(n=30)$, these results contradicted the idea that signet ring cells were insensitive to radiotherapy. ${ }^{20}$ Further studies are, therefore, needed to clarify this issue.

The NCCN guideline suggests that younger age is a highrisk factor for recurrence of LAGC. ${ }^{5}$ In a clinical setting, patients diagnosed at an earlier age generally exhibit more aggressive cancer behavior. ${ }^{21}$ Our study was consistent with this result, showing patients younger than 40 years may not benefit from adjuvant chemoradiotherapy chemotherapy.

Although the retrospective nonrandomized nature of this study meant we could not draw any firm conclusions, the available data found no significant differences between the two groups in terms of Lauren classification status, degree of differentiation, and venous or neural invasion. Despite the possibility of some bias originating from the limited sample size, the results suggesting that patients with signet ring cell carcinoma and mucinous adenocarcinoma might notably benefit from chemoradiotherapy needed to be further clarified. The proportions of patients receiving triplet and single regimen were small, and the results and significance of such subgroup analysis remain further explored. Some patients seek palliative treatment after recurrence, which may affect the OS.

\section{Conclusions}

In this study, we found that there was a significant 5- and 7-year OS benefits for patients who had adjuvant chemoradiotherapy as compared with adjuvant chemotherapy. Our subset analysis demonstrated that adjuvant chemoradiotherapy could improve the OS of patients with pIIIA and pIIIB stage disease and $\mathrm{LN}$ ratio $<50 \%$, but not in those with $\mathrm{pN} 3 \mathrm{~b}$ classification, tumor deposits and those undergoing total gastrectomy. We hope that these results may help to better guide the individualized treatments in patients with stage III LAGC after D2 gastrectomy.

\section{Acknowledgments}

This study was supported by the Youth Program of National Natural Science Foundation of China (Grant number 81502005) and the Natural Science Foundation of Shanghai (Grant number 15411961900). We thank Liwen Bianji, Edanz Group China, for editing the English text of drafts of this manuscript.

\section{Disclosure}

The authors report no conflicts of interest in this work.

\section{References}

1. Chen W, Zheng R, Baade PD, et al. Cancer statistics in China, 2015. CA Cancer J Clin. 2016;66(2):115-132. doi:10.3322/caac.21338

2. Sasako M, Sakuramoto S, Katai H, et al. Five-year outcomes of a randomized phase III trial comparing adjuvant chemotherapy with S-1 versus surgery alone in stage II or III gastric cancer. J Clin Oncol. 2011;29(33):4387-4393. doi:10.1200/JCO.2011.36.5908

3. Noh SH, Park SR, Yang HK, et al. Adjuvant capecitabine plus oxaliplatin for gastric cancer after D2 gastrectomy (CLASSIC): 5-year follow-up of an open-label, randomised phase 3 trial. Lancet Oncol. 2014;15(12):1389-1396. doi:10.1016/S1470-2045(14)70473-5

4. Smalley SR, Benedetti JK, Haller DG, et al. Updated analysis of SWOG-directed intergroup study 0116: a phase III trial of adjuvant radiochemotherapy versus observation after curative gastric cancer resection. J Clin Oncol. 2012;30(19):2327-2333. doi:10.1200/ JCO.2011.36.7136

5. National Comprehensive Cancer Network. NCCN Clinical Practice Guidelines in Oncology. Gastric Cancer. Version 1. 2018. Available from: https://www.nccn.org/professionals/physician_gls/f_guide lines.asp. Accessed June 04,2019.

6. Stumpf PK, Amini A, Jones BL, et al. Adjuvant radiotherapy improves overall survival in patients with resected gastric adenocarcinoma: a national cancer data base analysis. Cancer-Am Cancer Soc. 2017;123(17):3402-3409.

7. Park SH, Sohn TS, Lee J, et al. Phase III trial to compare adjuvant chemotherapy with capecitabine and cisplatin versus concurrent chemoradiotherapy in gastric cancer: final report of the adjuvant chemoradiotherapy in stomach tumors trial, including survival and subset analyses. J Clin Oncol. 2015;33(28):3130-3136. doi:10.1200/ JCO.2014.58.3930.

8. Wang X, Zhao DB, Yang L, et al. S-1 chemotherapy and intensity-modulated radiotherapy after D1/D2 lymph node dissection in patients with node-positive gastric cancer: a phase I/II study. $\mathrm{Br}$ J Cancer. 2018;118(3):338-343. doi:10.1038/bjc.2017.424

9. Ejaz A, Spolverato G, Kim Y, et al. Impact of external-beam radiation therapy on outcomes among patients with resected gastric cancer: a multi-institutional analysis. Ann Surg Oncol. 2014;21 (11):3412-3421. doi:10.1245/s10434-014-3776-5

10. Park SH, Sohn TS, Lee J, et al. Phase III trial to compare adjuvant chemotherapy with capecitabine and cisplatin versus concurrent chemoradiotherapy in gastric cancer: final report of the adjuvant chemoradiotherapy in stomach tumors trial, including survival and subset analyses. J Clin Oncol. 2015;33(28):3130. doi:10.1200/JCO.2014.58.3930

11. Ohri N, Garg MK, Aparo S, et al. Who benefits from adjuvant radiation therapy for gastric cancer? A meta-analysis. Int $J$ Radiat Oncol Biol Phys. 2013;86(2):330-335. doi:10.1016/j. ijrobp.2013.02.008

12. Wang MJ, Li C, Sun Y, Shen FJ, Wang CB. Prognostic effect of adjuvant chemoradiotherapy for patients with gastric cancer: an updated evidence of randomized controlled trials. Oncotarget. 2017;8(61):102880-102887. doi:10.18632/oncotarget.21983

13. Lee J, Lim DH, Kim S, et al. Phase III trial comparing capecitabine plus cisplatin versus capecitabine plus cisplatin with concurrent capecitabine radiotherapy in completely resected gastric cancer with D2 lymph node dissection: the ARTIST trial. J Clin Oncol. 2012;30 (3):268-273. doi:10.1200/JCO.2011.39.1953

14. Fan M, Li G, Shen L, Zhang H, Liang L, Zhang Z. Identification of patients with lymph node metastasis from gastric cancer who may benefit from adjuvant chemoradiotherapy after D2 dissection-do N3 patients benefit from additional radiation? Br J Radiol. 2016;89 (1059):20150758. doi:10.1259/bjr.20150758 
15. Cats A, Jansen E, van Grieken N, et al. Chemotherapy versus chemoradiotherapy after surgery and preoperative chemotherapy for resectable gastric cancer (CRITICS): an international, open-label, randomised phase 3 trial. Lancet Oncol. 2018;19(5):616-628. doi:10.1016/S1470-2045(18)30132-3

16. Amin M, Edge S, Greene F, Byrd D, Brookland R, Washington M. AJCC Cancer Staging Manual. Eighth ed. Springer; 2017.

17. Yildiz B, Etiz D, Dal P, et al. Tumor deposits: prognostic significance in gastric cancer patients. J Buon. 2016;21(6):1476-1481.

18. Lee HS, Lee HE, Yang HK, Kim WH. Perigastric tumor deposits in primary gastric cancer: implications for patient prognosis and staging. Ann Surg Oncol. 2013;20(5):1604-1613. doi:10.1245/ s10434-012-2692-9
19. Sun Z, Wang ZN, Xu YY, et al. Prognostic significance of tumor deposits in gastric cancer patients who underwent radical surgery. Surgery. 2012;151(6):871-881. doi:10.1016/j.surg.2011.12.027

20. Charalampakis N, Nogueras GG, Elimova E, et al. The proportion of signet ring cell component in patients with localized gastric adenocarcinoma correlates with the degree of response to pre-operative chemoradiation. Oncology. 2016;90(5):239-247.

21. Liu S, Feng F, Xu G, et al. Clinicopathological features and prognosis of gastric cancer in young patients. BMC Cancer. 2016;16:478. doi:10.1186/s12885-016-2489-5

\section{Publish your work in this journal}

Cancer Management and Research is an international, peer-reviewed open access journal focusing on cancer research and the optimal use of preventative and integrated treatment interventions to achieve improved outcomes, enhanced survival and quality of life for the cancer patient.
The manuscript management system is completely online and includes a very quick and fair peer-review system, which is all easy to use. Visit http://www.dovepress.com/testimonials.php to read real quotes from published authors. 